Pacific

Journal of

Mathematics

LOWER BOUNDARY HYPERPLANES OF THE CANONICAL LEFT CELLS IN THE AFFINE WEYL GROUP $W_{a}\left(\tilde{A}_{n-1}\right)$

JIAN-YI SHI 


\title{
LOWER BOUNDARY HYPERPLANES OF THE CANONICAL LEFT CELLS IN THE AFFINE WEYL GROUP $W_{a}\left(\tilde{A}_{n-1}\right)$
}

\author{
JIAN-YI SHI
}

Dedicated to Professor George Lusztig on his sixtieth birthday.

\begin{abstract}
Let $\Gamma$ be any canonical left cell of the affine Weyl group $W_{a}$ of type $\tilde{A}_{n-1}$ for $n>1$. We describe the lower boundary hyperplanes for $\Gamma$, answering two questions of Humphreys.
\end{abstract}

Let $W_{a}$ be an affine Weyl group and let $\Phi$ be the root system of the corresponding Weyl group. Fix a positive root system $\Phi^{+}$of $\Phi$. There is a bijection from $W_{a}$ to the set of alcoves in the euclidean space $E$ spanned by $\Phi$. We identify the elements of $W_{a}$ with the alcoves (also with the topological closure of the alcoves) of $E$. According to a result of Lusztig and Xi [1988], we know that the intersection of any two-sided cell of $W_{a}$ with the dominant chamber of $E$ is exactly a single left cell of $W_{a}$, called a canonical left cell. When $W_{a}$ is of type $\tilde{A}_{n-1}$, with $n>1$, there is a bijection $\phi$ from the set of two-sided cells of $W_{a}$ to the set of partitions of $n$; see Remark 2.1 and subsequent paragraphs, as well as [Shi 1986].

From now on, unless otherwise specified, we always assume that $W_{a}$ is an affine Weyl group of type $\tilde{A}_{n-1}$, where $n>1$. This article answers two questions posed recently by J. E. Humphreys (private communication):

(1) Can one find the set $B(L)$ of all the lower boundary hyperplanes for any canonical left cell $L$ of $W_{a}$ ?

(2) How does the partition $\phi(L)$ determine the set $B(L)$, and in which case does the set $B(L)$ determine the partition $\phi(L)$ also?

In the first two sections, we collect some concepts and known results for later use. In Section 3, we give criteria for a hyperplane to be the lower boundary of a canonical left cell of $W_{a}$. Then we prove our main results in Section 4 .

MSC2000: 20F55.

Keywords: affine Weyl groups, canonical left cells, hyperplanes, alcoves, sign types.

Supported by the NSF of China, the SF of the University Doctoral Program of ME of China, the Shanghai Priority Academic Discipline, and the CST of Shanghai (No. 03JC14027). 


\section{Sign types}

Let $\boldsymbol{n}=\{1,2, \ldots, n\}$ for $n \in \mathbb{N}$. An $\boldsymbol{n}$-sign type (or just a sign type) is by definition a matrix $X=\left(X_{i j}\right)_{i, j \in n}$ over the symbol set $\{+, \bigcirc,-\}$, with

$$
\left\{X_{i j}, X_{j i}\right\} \in\{\{+,-\},\{\bigcirc, \bigcirc\}\} \quad \text { for } \quad i, j \in \boldsymbol{n} .
$$

$X$ is determined entirely by its "upper unitriangular" part $X^{\Delta}=\left(X_{i j}\right)_{i<j}$. We identify $X$ with $X^{\Delta}$. $X$ is dominant, if $X_{i j} \in\{+, \bigcirc\}$ for any $i<j$ in $\boldsymbol{n}$, and is admissible, if

$$
\begin{aligned}
& -\in\left\{X_{i j}, X_{j k}\right\} \Longrightarrow X_{i k} \leqslant \max \left\{X_{i j}, X_{j k}\right\}, \\
& -\notin\left\{X_{i j}, X_{j k}\right\} \Longrightarrow X_{i k} \geqslant \max \left\{X_{i j}, X_{j k}\right\}
\end{aligned}
$$

for any $i<j<k$ in $\boldsymbol{n}$, where we set a total ordering: $-<\bigcirc<+$.

Lemma 1.1 ([Shi 1987b, Lemma 3.1; Shi 1999, Corollary 2.8]). (1) A dominant sign type $X=\left(X_{i j}\right)$ is admissible if and only iffor any $i \leqslant h<k \leqslant j$, condition $X_{i j}=\bigcirc$ implies $X_{h k}=\bigcirc$.

(2) If an admissible sign type $X=\left(X_{i j}\right)$ is not dominant, then there exists at least one $k$ with $1 \leqslant k<n$ and $X_{k, k+1}=-$.

Proof. This is an easy consequence of conditions (1-1).

Let $E=\left\{\left(a_{1}, \ldots, a_{n}\right) \in \mathbb{R}^{n} \mid \sum_{i=1}^{n} a_{i}=0\right\}$. This is a euclidean space of dimension $n-1$ with inner product $\left\langle\left(a_{1}, \ldots, a_{n}\right),\left(b_{1}, \ldots, b_{n}\right)\right\rangle=\sum_{i=1}^{n} a_{i} b_{i}$. For $i \neq j$ in $\boldsymbol{n}$, let $\alpha_{i j}=(0, \ldots, 0,1,0, \ldots, 0,-1,0, \ldots, 0)$, with 1 and -1 at the $i$-th and $j$-th positions, respectively. Then $\Phi=\left\{\alpha_{i j} \mid 1 \leqslant i \neq j \leqslant n\right\}$ is the root system of type $A_{n-1}$, which spans $E . \Phi^{+}=\left\{\alpha_{i j} \in \Phi \mid i<j\right\}$ is a positive root system of $\Phi$ with corresponding simple root system $\Pi=\left\{\alpha_{i, i+1} \mid 1 \leqslant i<n\right\}$. For any $\epsilon \in \mathbb{Z}$ and $i<j$ in $\boldsymbol{n}$, define a hyperplane

$$
H_{i j ; \epsilon}=\left\{\left(a_{1}, \ldots, a_{n}\right) \in E \mid a_{i}-a_{j}=\epsilon\right\} .
$$

Encode a connected component $C$ of $E \backslash \bigcup_{1 \leqslant i<j \leqslant n, \epsilon \in\{0,1\}} H_{i j ; \epsilon}$ by a sign type $X=\left(X_{i j}\right)_{i<j}$ as follows. Take any $v=\left(a_{1}, \ldots, a_{n}\right) \in C$ and, for $i<j$ in $\boldsymbol{n}$, set

$$
X_{i j}= \begin{cases}+ & \text { if } a_{i}-a_{j}>1, \\ - & \text { if } a_{i}-a_{j}<0, \\ \bigcirc & \text { if } 0<a_{i}-a_{j}<1 .\end{cases}
$$

$X$ only depends on $C$, but not on the choice of $v$; see [Shi 1986, Chapter 5]. Note that not all sign types can be obtained in this way.

Proposition 1.2 ([Shi 1986, Proposition 7.1.1 and §2]). A sign type $X=\left(X_{i j}\right)$ can be obtained in the above way if and only if it is admissible. 
Lemma 1.3. Let $X=\left(X_{i j}\right)$ be a dominant admissible sign type with $X_{p, p+1}=0$ for some $p$ with $1 \leqslant p<n$. Let $X^{\prime}=\left(X_{i j}^{\prime}\right)$ be the sign type given by

$$
X_{i j}^{\prime}= \begin{cases}X_{i j} & \text { if }(i, j) \neq(p, p+1), \\ - & \text { if }(i, j)=(p, p+1)\end{cases}
$$

for $i<j$ in $\boldsymbol{n}$. Then $X^{\prime}$ is admissible if and only if $X_{p h}=X_{p+1, h}$ for all $h \in \boldsymbol{n}$.

Proof. This is an easy consequence of (1-1).

For $\alpha \in \Phi$, let $s_{\alpha}$ be the reflection in $\alpha$ :

$$
s_{\alpha}(v)=v-2 \frac{\langle v, \alpha\rangle}{\langle\alpha, \alpha\rangle} \alpha .
$$

Let $T_{\alpha}$ be the translation by $\alpha$ : $T(v)=v+\alpha$. Define $s_{i}=s_{\alpha_{i, i+1}}$ for $1 \leqslant i<n$, and $s_{0}=T_{\alpha_{1 n}} s_{\alpha_{1 n}}$. Then $S=\left\{s_{i} \mid 0 \leqslant i<n\right\}$ forms a distinguished generator set of the affine Weyl group $W_{a}$ of type $\tilde{A}_{n-1}$.

A connected component in

$$
E \backslash \bigcup_{\substack{1 \leqslant i<j \leqslant n \\ k \in \mathbb{Z}}} H_{i j ; k}
$$

is called an alcove. The (right) action of $W_{a}$ on $E$ induces a simply transitive permutation on the set $\mathfrak{A}$ of alcoves in $E$. There exists a bijection $w \mapsto A_{w}$ from $W_{a}$ to $\mathfrak{A}$ such that $A_{1}$ (where 1 is the identity element of $W_{a}$ ) is the unique alcove in the dominant chamber of $E$ whose closure contains the origin and such that $\left(A_{y}\right) w=A_{y w}$ for $y, w \in W_{a}$; see [Shi 1987a, Proposition 4.2]. To each $w \in W_{a}$ we associate an admissible sign type $X(w)$ that contains the alcove $A_{w}$. An admissible sign type $X$ can be identified with the set $\left\{w \in W_{a} \mid X(w)=X\right\}$.

\section{Partitions and Kazhdan-Luzstig cells}

Let $(P, \preceq)$ be a finite poset. By a chain of $P$, we mean a totally ordered subset of $P$ (allow to be an empty set). Also, a cochain of $P$ is a subset $K$ of $P$ whose elements are pairwise incomparable. A $k$-(co)chain family in $P(k \geqslant 1)$ is a subset $J$ of $P$ which is a disjoint union of $k$ (co)chains $J_{i}(1 \leqslant i \leqslant k)$. We usually write $J=J_{1} \cup \cdots \cup J_{k}$.

A partition of $n \in \mathbb{N}$ is a sequence $\lambda=\left(\lambda_{1}, \lambda_{2}, \ldots, \lambda_{r}\right)$ of positive integers such that $\lambda_{1} \geqslant \lambda_{2} \geqslant \cdots \geqslant \lambda_{r}$ and $\sum_{i=1}^{r} \lambda_{i}=n$. In particular, when $\lambda_{1}=\cdots=\lambda_{r}=a$, we also write $\lambda=\left(a^{r}\right)$, and call it a rectangular partition. Let $\Lambda_{n}$ be the set of all partitions of $n$.

Let $\lambda=\left(\lambda_{1}, \lambda_{2}, \ldots, \lambda_{r}\right)$ and $\mu=\left(\mu_{1}, \mu_{2}, \ldots, \mu_{t}\right)$ be in $\Lambda_{n}$. Write $\lambda \leqslant \mu$ if the inequalities $\sum_{j=1}^{i} \lambda_{j} \leqslant \sum_{j=1}^{i} \mu_{j}$ hold for $i \geqslant 1$. We say that $\mu$ is conjugate to $\lambda$ 
if $\mu_{i}=\left|\left\{j \mid \lambda_{j} \geqslant i, 1 \leqslant j \leqslant r\right\}\right|$ for $1 \leqslant i \leqslant t$, where $|X|$ stands for the cardinality of the set $X$.

Let $d_{k}$ be the maximal cardinality of a $k$-chain family in $P$ for $k \geqslant 1$. Then $d_{1}<d_{2}<\cdots<d_{r}=n=|P|$ for some $r \geqslant 1$. Let $\lambda_{1}=d_{1}$ and $\lambda_{i}=d_{i}-d_{i-1}$ for $1<i \leqslant r$. Then $\lambda_{1} \geqslant \lambda_{2} \geqslant \cdots \geqslant \lambda_{r}>0$ by [Greene 1976, Theorem 1.6]. We get $\phi(P)=\left(\lambda_{1}, \ldots, \lambda_{r}\right) \in \Lambda_{n}$, called the partition associated to chains in $P$. Replacing the word " $k$-chain family" by " $k$-cochain family", we can also define $\psi(P)=\left(\mu_{1}, \ldots, \mu_{t}\right) \in \Lambda_{n}$, again by [Greene 1976, Theorem 1.6], called the partition associated to cochains in $P$. Moreover, $\psi(P)$ is conjugate to $\phi(P)$.

Remark 2.1. Let $(P, \preceq)$ be a finite poset with $\psi(P)=\left(\mu_{1}, \ldots, \mu_{t}\right)$. For $1 \leqslant k \leqslant t$, let $P^{(k)}=P_{1} \cup \cdots \cup P_{k}$ be a $k$-cochain family of $P$ with $\left|P^{(k)}\right|=\sum_{h=1}^{k} \mu_{h}$. Then $\mu_{1} \geqslant\left|P_{i}\right| \geqslant \mu_{k}$ for $1 \leqslant i \leqslant k$. In particular, when $\psi(P)=\left(a^{t}\right)$ is rectangular, we have $\left|P_{1}\right|=\cdots=\left|P_{k}\right|=a$. This fact will be used in the proof of Lemma 4.2.

For each admissible sign type $X=\left(X_{i j}\right)$, we write $i \leqslant_{X} j$ in $\boldsymbol{n}$ if either $i=j$ or $X_{i j}=+$. By [Shi 1999, Lemma 2.2], the order $\leqslant_{X}$ is a partial order on $\boldsymbol{n}$. We associate to $X$ two partitions $\phi(X)$ and $\psi(X)$ of $n$ defined above.

Kazhdan and Lusztig [1979] defined certain equivalence classes in a Coxeter system $(W, S)$, called a left cell, a right cell and a two-sided cell.

Let $W_{a}$ be the affine Weyl group of type $\tilde{A}_{n-1}$ for $n>1$. Each element $w$ of $W_{a}$ determines a sign type $X(w)$, and hence it in turn determines two partitions $\phi(w):=\phi(X(w))$ and $\psi(w):=\psi(X(w))$. This defines two maps $\phi, \psi: W_{a} \longrightarrow$ $\Lambda_{n}$, each of which induces, by [Shi 1986, Theorem 17.4], a bijection from the set of two-sided cells of $W_{a}$ to the set $\Lambda_{n}$.

To each $w \in W_{a}$, we associate a set $\mathscr{R}(w)=\{s \in S \mid w s<w\}$, where $\leqslant$ is the Bruhat order in the Coxeter system $\left(W_{a}, S\right)$. Define

$$
Y_{0}=\left\{w \in W_{a} \mid \mathscr{R}(w) \subseteq\left\{s_{0}\right\}\right\} .
$$

By [Lusztig and Xi 1988, Theorem 1.2], the intersection of $Y_{0}$ with any two-sided cell $\phi^{-1}(\lambda)\left(\lambda \in \Lambda_{n}\right)$ is a single left cell of $W_{a}$, written $\Gamma_{\lambda}$ and called a canonical left cell.

\section{Lower boundary of a canonical left cell}

We now define a lower boundary hyperplane for any $F \subset W_{a}$, and give criteria for a hyperplane of $E$ to be lower boundary for a canonical left cell of $W_{a}$.

For $i<j$ in $\boldsymbol{n}$ and $k \in \mathbb{Z}$, the hyperplane $H_{i j ; k}$ divides the space $E$ into three parts: $H_{i j ; k}^{+}=\left\{v \in E \mid\left\langle v, \alpha_{i j}\right\rangle>k\right\}, H_{i j ; k}^{-}=\left\{v \in E \mid\left\langle v, \alpha_{i j}\right\rangle<k\right\}$, and $H_{i j ; k}$. For any set $F$ of alcoves in $E$, call $H_{i j ; k}$ a lower boundary hyperplane of $F$ if $\bigcup_{A \in F} A \subset H_{i j ; k}^{+}$and if there exists some alcove $C$ in $F$ such that $\bar{C} \cap H_{i j ; k}$ is a 
facet of $C$ of dimension $n-2$, where $\bar{C}$ stands for the closure of $C$ in $E$ under the usual topology.

Let $\Gamma$ be a canonical left cell of $W_{a}$. As a subset in $W_{a}, \Gamma$ is a union of some dominant sign types, by [Shi 1986, Proposition 18.2.2]; denote by $S(\Gamma)$ the set of these sign types. Regarded as a union of alcoves, the topological closure of $\Gamma$ in $E$ is connected [Shi 1986, Theorem18.2.1] and is bounded by a certain set of hyperplanes in $E$ of the form $H_{i j ; \epsilon}$, for $1 \leqslant i<j \leqslant n$ and $\epsilon=0,1$, defined in (1-2). Then a lower boundary hyperplane of $\Gamma$ must be one of such hyperplanes. Let $B(\Gamma)$ be the set of all the lower boundary hyperplanes of $\Gamma$. Given a hyperplane $H_{i j ; \epsilon}$ with $1 \leqslant i<j \leqslant n$ and $\epsilon=0,1$, we see that $H_{i j ; \epsilon} \in B(\Gamma)$ if and only if one of the following conditions holds.

Condition 3.1. $\epsilon=1, X_{i j}=+$ for all $X=\left(X_{a b}\right) \in S(\Gamma)$, and there exists some $Y=\left(Y_{a b}\right) \in S(\Gamma)$ such that the sign type $Y^{\prime}=\left(Y_{a b}^{\prime}\right)$ defined below is admissible:

$$
Y_{a b}^{\prime}= \begin{cases}Y_{a b} & \text { if }(a, b) \neq(i, j), \\ \bigcirc & \text { if }(a, b)=(i, j) .\end{cases}
$$

Condition 3.2. $\epsilon=0$, and there exists some $X=\left(X_{a b}\right) \in S(\Gamma)$ with $X_{i j}=\bigcirc$ such that the sign type $X^{\prime}=\left(X_{a b}^{\prime}\right)$ defined by

$$
X_{a b}^{\prime}= \begin{cases}X_{a b} & \text { if }(a, b) \neq(i, j) \\ - & \text { if }(a, b)=(i, j)\end{cases}
$$

is admissible.

Remark 3.3. By Lemma 1.1(2), Condition 3.2 happens only if $j=i+1$.

Proposition 3.4. (1) $H_{i, i+1 ; 0} \in B(\Gamma)$ if and only if there exists some $X=\left(X_{a b}\right) \in$ $S(\Gamma)$ such that $X_{i, h}=X_{i+1, h}$ for all $h \in \boldsymbol{n}$. In particular, when these conditions hold, we have $X_{i, i+1}=\bigcirc$.

(2) If $H_{i j ; 1} \in B(\Gamma)$ and if either $i \leqslant k<l \leqslant j$ or $k \leqslant i<j \leqslant l$, then $H_{k l ; 1} \in B(\Gamma)$ if and only if $(i, j)=(k, l)$.

Proof. Part (1) follows from Condition 3.2 and Lemma 1.3. Then part (2) is a direct consequence of Condition 3.1 and Lemma 1.1(1).

\section{Description of the sets $B_{0}\left(\Gamma_{\lambda}\right)$ and $B_{1}\left(\Gamma_{\lambda}\right)$}

We now answer the two questions of Humphreys.

Let $\Gamma_{\lambda}$ be the canonical left cell of $W_{a}$ corresponding to $\lambda \in \Lambda_{n}$. Let $B_{\epsilon}\left(\Gamma_{\lambda}\right)=$ $\left\{H_{i j ; \epsilon} \mid H_{i j ; \epsilon} \in B\left(\Gamma_{\lambda}\right)\right\}$ for $\epsilon=0,1$.

Lemma 4.1. Suppose that $\lambda=\left(\lambda_{1}, \ldots, \lambda_{r}\right) \in \Lambda_{n}$ contains at least two different parts. Then $B_{0}\left(\Gamma_{\lambda}\right)=\left\{H_{i, i+1 ; 0} \mid 1 \leqslant i<n\right\}$. 
Proof. Let $\mu=\left(\mu_{1}, \ldots, \mu_{t}\right)$ be the conjugate partition of $\lambda$. Then $\mu$ also contains at least two different parts. Given any $p$ with $1 \leqslant p<n$, there exists a permutation $a_{1}, a_{2}, \ldots, a_{t}$ of $1,2, \ldots, t$ such that $m_{s}<p$ and $m_{s+1}>p$ for some $s$ with $0 \leqslant s<t$, where $m_{u}:=\sum_{k=1}^{u} \mu_{a_{k}}$ for $0 \leqslant u \leqslant t$ with the convention that $m_{0}=0$. Define a dominant sign type $X=\left(X_{i j}\right)$ such that for any $i, j$ with $1 \leqslant i<j \leqslant n$, $X_{i j}=\bigcirc$ if and only if $m_{h}<i<j \leqslant m_{h+1}$ for some $h$ with $0 \leqslant h<t$. Clearly, $X$ is admissible with $\psi(X)=\mu$. Hence $X \in S\left(\Gamma_{\lambda}\right)$. We see also that $X_{p h}=X_{p+1, h}$ for all $h$ such that $1 \leqslant h \leqslant n$. So we conclude that $H_{p, p+1 ; 0} \in B_{0}\left(\Gamma_{\lambda}\right)$ by Proposition 3.4(1). Our result follows by Remark 3.3.

Lemma 4.2. For a rectangular partition $\left(k^{a}\right) \in \Lambda$ with $a, k \in \mathbb{N}$, we have

$$
B_{0}\left(\Gamma_{\left(k^{a}\right)}\right)=\left\{H_{p, p+1 ; 0} \mid 1 \leqslant p<n, a \nmid p\right\} .
$$

Proof. Let $X=\left(X_{i j}\right)$ be a dominant admissible sign type. Then a maximal cochain in $\boldsymbol{n}$ with respect to $\leqslant_{X}$ must consist of consecutive numbers. Now suppose $\psi(X)=\left(a^{k}\right)$. Then by Remark 2.1 , we can take a maximal $k$-cochain family $\boldsymbol{n}=P_{1} \cup \cdots \cup P_{k}$ such that $P_{h}=\{a(h-1)+1, a(h-1)+2, \ldots, a h\}$ with $1 \leqslant h \leqslant k$ are the maximal cochains in $\boldsymbol{n}$ with respect to $\leqslant_{X}$. We have $X_{a(h-1)+1, a h}=0$ and $X_{a(h-1)+1, a h+1}=+$, which are different. So by the arbitrariness of $X$ and by Proposition 3.4(1), we see that

$$
H_{a h, a h+1 ; 0} \notin B_{0}\left(\Gamma_{\left(k^{a}\right)}\right) \quad \text { for } 1 \leqslant h<k .
$$

On the other hand, let $Y=\left(Y_{i j}\right)$ be a sign type defined by

$$
Y_{i j}= \begin{cases}\bigcirc & \text { if } a(h-1)<i<j \leqslant a h \text { for some } 1 \leqslant h \leqslant k, \\ + & \text { otherwise }\end{cases}
$$

for $1 \leqslant i<j \leqslant n$. Then it is clear that $Y$ is dominant admissible with $\psi(Y)=\left(a^{k}\right)$. Suppose $a(h-1)<p<a h$ for some $h \in[1, k]$. Then $Y_{p, p+1}=\bigcirc$. We see also that $Y_{p h}=Y_{p+1, h}$ for all $h \in[1, n]$. By Proposition 3.4(1), we have

$$
H_{p, p+1 ; 0} \in B_{0}\left(\Gamma_{\left(k^{a}\right)}\right) \quad \text { for all } p \text { with } 1 \leqslant p<n \text { and } a \nmid p .
$$

The result follows from this, (4-1), and Remark 3.3.

Theorem 4.3. $B_{0}\left(\Gamma_{\lambda}\right)=\left\{H_{i, i+1 ; 0} \mid 1 \leqslant i<n\right\}$ for all $\lambda \in \Lambda_{n}$ unless $\lambda$ is a rectangular partition. In the latter case, say $\lambda=\left(k^{a}\right)$ for $k, a \in \mathbb{N}$, we have $B_{0}\left(\Gamma_{\left(k^{a}\right)}\right)=$ $\left\{H_{p, p+1 ; 0} \mid 1 \leqslant p<n, a \nmid p\right\}$.

Proof. We see that a partition is nonrectangular if and only if it contains at least two different parts. So our result follows immediately from Lemmas 4.1 and 4.2.

Theorem 4.4. $B_{1}\left(\Gamma_{\lambda}\right)=\left\{H_{i, i+r ; 1} \mid 1 \leqslant i \leqslant n-r\right\}$ for $\lambda=\left(\lambda_{1}, \ldots, \lambda_{r}\right) \in \Lambda_{n}$. 
Proof. Let $\mu=\left(\mu_{1}, \ldots, \mu_{t}\right) \in \Lambda_{n}$ be conjugate to $\lambda$. First we claim that, for any $X=\left(X_{i j}\right) \in S\left(\Gamma_{\lambda}\right)$,

$$
X_{i, i+r}=+ \text { for } i=1, \ldots, n-r .
$$

Otherwise, there would exist some $X=\left(X_{i j}\right) \in S\left(\Gamma_{\lambda}\right)$ with $X_{i, i+r}=\bigcirc$ for some $i$, $1 \leqslant i \leqslant n-r$. By Lemma 1.1(1), we would have $X_{h k}=\bigcirc$ for all $h, k$ such that $i \leqslant h<k \leqslant i+r$. Then $\{i, i+1, \ldots, i+r\}$ would be a cochain in $n$ with respect to the partial order $\leqslant_{X}$, whose cardinality is $r+1>\mu_{1}=r$, contradicting the assumption $\psi\left(\Gamma_{\lambda}\right)=\left(\mu_{1}, \mu_{2}, \ldots, \mu_{t}\right)$.

Next we want to find, for any $p$ with $1 \leqslant p \leqslant n-r$, some $Y=\left(Y_{i j}\right) \in S\left(\Gamma_{\lambda}\right)$ such that the sign type $Y^{\prime}=\left(Y_{i j}^{\prime}\right)$ defined by

$$
Y_{i j}^{\prime}= \begin{cases}Y_{i j} & \text { if }(i, j) \neq(p, p+r), \\ \bigcirc & \text { if }(i, j)=(p, p+r)\end{cases}
$$

for $1 \leqslant i<j \leqslant n$, is admissible. If this happens, we automatically have $\psi\left(Y^{\prime}\right) \supsetneqq \mu$ by the proof of (4-2).

Take a permutation $a_{1}, a_{2}, \ldots, a_{t}$ of $1,2, \ldots, t$ satisfying two conditions:

(1) Let $m_{u}=\sum_{k=1}^{u} \mu_{a_{k}}$ for $0 \leqslant u \leqslant t$ with the convention that $m_{0}=0$. Then there exists some $s \in[0, t)$ such that $a_{s+1}=1, m_{s}<p$ and $m_{s+1} \geqslant p$.

(2) $s$ is the largest possible number with the property (1) when $a_{1}, a_{2}, \ldots, a_{t}$ ranges over all the permutations of $1,2, \ldots, t$.

Then we have $t-s \geqslant 2, p \leqslant m_{s+1}<p+r$ and $m_{s+2} \geqslant p+r$. Define a dominant sign type $Y=\left(Y_{i j}\right)$ such that $Y_{i j}=\bigcirc$ if and only if either

$m_{u}<i<j \leqslant m_{u+1}$ for $0 \leqslant u<t, \quad$ or $\quad p \leqslant i<j \leqslant p+r$ with $(i, j) \neq(p, p+r)$.

By Lemma 1.1(1), $Y$ is admissible with $\psi(Y)=\mu$, i.e., $Y \in S\left(\Gamma_{\lambda}\right)$. Clearly, the sign type $Y^{\prime}$ obtained from $Y$ as in (4-3) is also dominant admissible by Lemma 1.1(1). This implies by Condition 3.1 that $H_{p, p+r ; 1}$ belongs to $B_{1}\left(\Gamma_{\lambda}\right)$ for any $p=1, \ldots, n-r$. The result follows by Proposition 3.4(2).

Remark 4.5. Theorems 4.3 and 4.4 answer the two questions of Humphreys. In particular, the canonical left cells of $W_{a}$ associated to the rectangular partitions are determined entirely by the corresponding $B_{1}$-set of hyperplanes. From the above description of $B_{0}$-sets of hyperplanes, we see that compared with the other canonical left cells of $W_{a}$, the positions of the canonical left cells associated to rectangular partitions are farther from the walls of the dominant chamber.

Remark 4.6. When $\lambda=(n)$, we have $B_{0}\left(\Gamma_{\lambda}\right)=\varnothing$ and

$$
B_{1}\left(\Gamma_{\lambda}\right)=\left\{H_{i, i+1 ; 1} \mid 1 \leqslant i<n\right\} .
$$


Actually, this is the unique canonical left cell whose $B_{1}$-set contains a hyperplane of the form $H_{i, i+1 ; 1}$. Also, this is the unique canonical left cell whose $B_{0}$-set is empty. On the other hand, $B_{0}\left(\Gamma_{\left(1^{n}\right)}\right)=\left\{H_{i, i+1 ; 0} \mid 1 \leqslant i<n\right\}$ and $B_{1}\left(\Gamma_{\left(1^{n}\right)}\right)=\varnothing$. $\Gamma_{\left(1^{n}\right)}$ is the unique canonical left cell whose $B_{1}$-set is empty.

Remark 4.7. When $n \in \mathbb{N}$ is a prime number, the $B_{0}$-sets of all the canonical left cells $\Gamma_{\lambda}$ of $W_{a}$ are $\left\{H_{i, i+1 ; 0} \mid 1 \leqslant i<n\right\}$, except for the case where $\lambda=(n)$.

Remark 4.8. Now assume that $\left(W_{a}, S\right)$ is an irreducible affine Weyl group of arbitrary type with $\Delta$ a choice of simple roots system of $\Phi$. We are unable to describe the lower boundary hyperplanes for a canonical left cell $L$ of $W_{a}$ in general. This is because $L$ is not always a union of some sign types (as in the case of type $\tilde{B}_{2}$ ). But we know that $L$ is a single sign type when $L$ is in either the lowest or the highest two-sided cell of $W_{a}$ (see [Shi 1987c; Shi 1988]) for which we can describe its lower boundary hyperplanes: if $L$ is in the lowest two-sided cell of $W_{a}$, then $B_{1}(L)=\left\{H_{\alpha ; 1} \mid \alpha \in \Delta\right\}$ and $B_{0}(L)=\varnothing$, where $H_{\alpha ; 1}:=\left\{v \in E \mid\left\langle v, \alpha^{\vee}\right\rangle=1\right\}$ and $\alpha^{\vee}=2 \alpha /\langle\alpha, \alpha\rangle$; if $L$ is in the highest two-sided cell of $W_{a}$, then $B_{1}(L)=\varnothing$ and $B_{0}(L)=\left\{H_{\alpha ; 0} \mid \alpha \in \Delta\right\}$. This extends the result in Remark 4.6. We conjecture that any canonical left cell of $W_{a}$ is a union of some sign types whenever $W_{a}$ has a simply-laced type, namely $\tilde{A}, \tilde{D}$ or $\tilde{E}$. If this is true, one would be able to describe the lower boundary hyperplanes for the canonical left cells of these groups.

\section{References}

[Greene 1976] C. Greene, "Some partitions associated with a partially ordered set", J. Combinatorial Theory Ser. A 20:1 (1976), 69-79. MR 53 \#2763 Zbl 0323.06002

[Kazhdan and Lusztig 1979] D. Kazhdan and G. Lusztig, "Representations of Coxeter groups and Hecke algebras”, Invent. Math. 53:2 (1979), 165-184. MR MR560412 (81j:20066) Zbl 0499.20035

[Lusztig and Xi 1988] G. Lusztig and N. H. Xi, "Canonical left cells in affine Weyl groups", Adv. in Math. 72:2 (1988), 284-288. MR 89m:17027 Zbl 0664.20028

[Shi 1986] J. Y. Shi, The Kazhdan-Lusztig cells in certain affine Weyl groups, Lecture Notes in Mathematics 1179, Springer, Berlin, 1986. MR 87i:20074 Zbl 0582.20030

[Shi 1987a] J. Y. Shi, "Alcoves corresponding to an affine Weyl group", J. London Math. Soc. (2) 35:1 (1987), 42-55. MR 88g:20103a Zbl 0681.20032

[Shi 1987b] J. Y. Shi, "Sign types corresponding to an affine Weyl group”, J. London Math. Soc. (2) 35:1 (1987), 56-74. MR 88g:20103b Zbl 0681.20033

[Shi 1987c] J. Y. Shi, “A two-sided cell in an affine Weyl group”, J. London Math. Soc. (2) 36:3 (1987), 407-420. MR 88k:20073 Zbl 0598.20045

[Shi 1988] J. Y. Shi, "A two-sided cell in an affine Weyl group. II”, J. London Math. Soc. (2) 37:2 (1988), 253-264. MR 89a:20055 Zbl 0613.20026

[Shi 1999] J. Y. Shi, “Sign types associated to posets", J. Combin. Theory Ser. A 88:1 (1999), 36-53. MR 2000i:05191 Zbl 0961.20037

Received November 21, 2004. 
JIAN-YI SHI

DEPARTMENT OF MATHEMATICS

EAST CHINA NORMAL UNIVERSITY

SHANGHAI, 200062

CHINA

and

CENTER FOR COMbinAtorics

NANKAI UNIVERSITY

TIANJIN, 300071

CHINA

jyshi@math.ecnu.edu.cn 